\title{
END EXTENSIONS, CONSERVATIVE EXTENSIONS, AND THE RUDIN-FROLÍK ORDERING
}

BY

\author{
ANDREAS BLASS( $(1)$
}

\begin{abstract}
The ordering of ultrafilters on the natural numbers defined by " $E$-prod $N$ is an end extension of $D$-prod $N$," the ordering defined by " $E$ prod $N$ is a conservative extension of $D$-prod $N$," and the Rudin-Frolik ordering are proved to be distinct if the continuum hypothesis holds. These three orderings are also characterized in terms of (not necessarily internal) ultrafilters in the Boolean algebra of internal sets of natural numbers in a nonstandard universe.
\end{abstract}

The purpose of this paper is to clarify the connections between three treelike orderings of the ultrafilters on the set $\omega$ of natural numbers. One of these orderings is the Rudin-Frolik ordering, studied in [4], [5], [12]. The other two orderings of ultrafilters are most easily described in terms of the ultrapowers of $\omega$ formed using the ultrafilters. One corresponds to the relation of end extension between ultrapowers, the other to the relation of conservative extension. (The definitions are given in detail below.) We use the notations $\leqslant_{R F}$, $\leqslant_{E}$, and $\leqslant_{C}$ for these three orderings. It is known that $D \leqslant_{R F} E$ implies $D \leqslant_{C} E$ which in turn implies $D \leqslant_{E} E$. We show assuming the continuum hypothesis that neither of these implications can be reversed. In the construction of counterexamples to the converse implications, it is convenient to use a certain natural correspondence between ultrafilters that map to a given ultrafilter $D$ and ultrafilters in the Boolean algebra $D$-prod $\mathscr{P}(\omega)$, where $\mathscr{P}$ means power set. This correspondence also leads to a rather elegant "uniform" description of the three orderings under consideration.

After reviewing definitions and preliminary results in $\$ 1$, we devote $\$ 2$ to the correspondence just mentioned and the characterizations of the three orderings using it. In $\$ 3$, we construct, for every nonprincipal ultrafilter $D$ on $\omega$, an ultrafilter $E$ such that $D \leqslant_{E} E$ but $D \aleph_{C} E$. In $\$ 4$, we construct, for every

Received by the editors September 24, 1975.

AMS (MOS) subject classifications (1970). Primary 02H20, 04A20.

Key words and phrases. Ultrafilter, ultrapower, nonstandard model, Rudin-Keisler order, RudinFrolik order, elementary extensions, end extensions, conservative extensions.

(1) Partially supported by NSF Contract MPS 74-12011. 
such $D$, an $E$ such that $D \leqslant_{C} E$ but $D \$_{R F} E$. Both of these constructions depend on the continuum hypothesis. The construction in $\$ 4$ also depends on the existence of an external unbounded subset of $D$-prod $\kappa_{1}$, all of whose initial segments determined by countable ordinals are internal; such a set is constructed (without any appeal to the continuum hypothesis) in $\$ 5$.

Propositions 1 and 4, part of Proposition 2, and Theorem 11 and its corollaries with "end extension" in place of "conservative extension" are taken from $\$ \$ 14$ and 22 of my thesis [1], written under the supervision of Frank Wattenberg for whose guidance and encouragement I here record my gratitude.

1. Definitions and preliminary results. Let $D$ be an ultrafilter on the set $\omega$ of natural numbers, and let $f$ map $\omega$ into some set $X$. The germ $[f]_{D}$ of $f$ modulo $D$ consists of all functions from $\omega$ to $X$ that agree with $f$ on some set in $D$. The ultrafilter $f(D)=\left\{A \subseteq X \mid f^{-1}(A) \in D\right\}$ depends only on $D$ and $[f]_{D}$; we call $[f]_{D}$ a morphism from $D$ to $f(D)$ and define composition of morphisms in the obvious way. A morphism $[f]_{D}$ is an isomorphism (i.e., it has a twosided inverse) iff $f$ is one-to-one on some set in $D$. The only morphism from an ultrafilter to itself is the germ of the identity map. The Rudin-Keisler ordering of ultrafilters on $\omega$ is defined by setting $D \leqslant_{R K} E$ iff there is a morphism from $E$ to $D$; this is a pre-order, and the associated equivalence relation is just isomorphism.

We fix a bijection between $\omega \times \omega$ and $\omega$, and we use it to identify these two sets; the two projections $p_{i}: \omega \times \omega \rightarrow \omega(i=1,2)$ are thus viewed as maps of $\omega$ onto itself. When considering a morphism $[f]_{D}: D \rightarrow E$, we of ten visualize $D$ as an ultrafilter on $\omega \times \omega, E$ as an ultrafilter on $\omega$, and the morphism as $\left[p_{1}\right]_{D}$. This involves no loss of generality, for any given morphism $[f]_{D}$ can be expressed as the composite of an isomorphism $D \cong D^{\prime}$ and a projection $\left[p_{1}\right]_{D^{\prime}}: D^{\prime} \rightarrow E$.

We shall be concerned with ultrapowers of two structures. One is $N$, the standard model of full arithmetic; its universe is $\omega$ and its relations and functions are all the relations and functions on $\omega$. The other is $V$, whose universe is a transitive set large enough to contain all the sets we need below $\left(R_{\mathrm{K}_{2}}\right.$ is big enough) and whose only relation is membership, $\in$. In connection with any nonstandard model ${ }^{*} V$ of $V$, with elementary embedding $x \rightarrow^{*} x$ of $V$ into ${ }^{*} V$, we use the customary terminology of nonstandard analysis. Thus, an element of ${ }^{*} V$ is standard if it is ${ }^{*} x$ for some $x \in V$. A subset of ${ }^{*} V$ is internal if it is the extension $\left\{y \in{ }^{*} V \mid y^{*} \in x\right\}$ of some $x \in{ }^{*} V$; otherwise it is external. We occasionally (as in the next sentence) fail to distinguish between an internal subset of ${ }^{*} V$ and the element of ${ }^{*} V$ whose extension it is. An internal subset of ${ }^{*} V$ is ${ }^{*}$ finite (resp. ${ }^{*}$ countable) if it satisfies in ${ }^{*} V$ the formula defining finiteness (resp. countability) in $V$, i.e., if it is in internal one- 
to-one correspondence with an element of ${ }^{*} \omega$ (resp. with ${ }^{*} \omega$ or one of its elements).

If ${ }^{*} N$ is a nonstandard model of $N$, its standard elements (resp. relations, functions) are those of the form ${ }^{*} x$ (resp. ${ }^{*} R,{ }^{*} f$ ) with $x \in \omega$ (resp. $R$ a relation on $\omega, f$ a function on $\omega)$. An $n$-place relation is parametrically definable if it has the form $\left\{\left.\mathbf{x} \in{ }^{*} N^{n}\right|^{*} R(\mathbf{x}, q)\right\}$ for some $(n+1)$-place relation $R$ on $\omega$ and some parameter $q \in{ }^{*} N$. Parametric definability of functions is defined similarly.

In any nonstandard model ${ }^{*} V$ of $V$, the extension of ${ }^{*} \omega$ and the standard relations and functions on it constitute a nonstandard model ${ }^{*} N$ of $N$. In this situation, any parametrically definable relation or function on ${ }^{*} N$ is internal in ${ }^{*} V$; the converse holds if ${ }^{*} V$ is $D$-prod $V$ (so ${ }^{*} N=D$-prod $N$ ) for some ultrafilter $D$ on $\omega$, but in general there may be relations on ${ }^{*} N$ that are internal in ${ }^{*} V$ without being parametrically definable in ${ }^{*} N$.

For any structure $A$, any morphism $[f]_{D}: D \rightarrow E$ of ultrafilters induces an elementary embedding of ultrapowers, $\hat{f}: E$-prod $A \rightarrow D$-prod $A$ defined by $\hat{f}\left([g]_{E}\right)=[g \circ f]_{D}$. Conversely, any elementary embedding $j: E$-prod $N$ $\rightarrow D-\operatorname{prod} N$, where $D$ and $E$ are ultrafilters on $\omega$, is of the form $\hat{f}$ for a unique $[f]_{D}: D \rightarrow E$, namely $j\left([\text { identity }]_{E}\right)$. Thus, there is a canonical bijection between morphisms of ultrafilters on $\omega$ and elementary embeddings of ultrapowers of $N$. We exploit this fact by defining some classes of morphisms in terms of properties of the corresponding embeddings.

We call $[f]_{D}: D \rightarrow E$ an end-extension morphism, or $E$-morphism for short, if $\hat{f}(E$-prod $N)$ is an initial segment of $D$-prod $N$ (with respect to the ordering $* \leqslant$ corresponding to the standard ordering of $\omega$ ). Using the definitions of ultrapowers and $\hat{f}$, we see that $[f]_{D}$ is an $E$-morphism iff every function $g: \omega \rightarrow \omega$ that is bounded on each of the fibers $f^{-1}\{n\}$ of $f$ is in fact constant on the intersection of these fibers with a certain set (independent of $n$ ) in $D$. (Briefly, fiberwise bounded functions are fiberwise constant modulo $D$.) We write $E \leqslant_{E} D$ to mean that there is an $E$-morphism from $D$ to $E$. As the composite of two $E$-morphisms is an $E$-morphism, we see that $\leqslant_{E}$ is a preorder, stronger than $\leqslant_{R K}$.

PROPOSITION 1. (a) Of any two E-morphisms with the same domain, one factors through the other by an E-morphism.

(b) Any two E-morphisms with the same domain and codomain are equal. That is, the category of E-morphisms is just the pre-order $\leqslant_{E}$.

(c) The pre-order $\leqslant_{E}$ is tree-like. That is, the predecessors of any point are linearly pre-ordered.

Proof. When (a) is translated into a statement about ultraproducts, it says that, of any two initial segments of the same model, one is an initial segment of the other; but that is obvious. For part (b), simply note that the 
factorization given by (a) must be by the identity, for there is no other morphism from the codomain ultrafilter to itself. Finally, (c) is just a reformulation of (a).

Let $N \prec A \prec B$ ( $\prec$ means elementary submodel). Following Phillips [10], we say that $B$ is a conservative extension of $A$ if every parametrically definable subset of $B$, when intersected with $A$, yields a parametrically definable subset of $A$. As Phillips noted, this implies that $A$ is an initial segment of $B$, for, if $b \in B-A$, then $\left\{x \in A \mid x^{*}<b\right.$ in $\left.B\right\}$ is a parametrically definable subset of $A$ containing 0 and closed under successor, so it must be all of $A$. Let us define $[f]_{D}: D \rightarrow E$ to be a conservative morphism, or $C$-morphism, if $D$-prod $N$ is a conservative extension of $\hat{f}(E$-prod $N)$. Then every $C$-morphism is an $E$ morphism. Since "conservative extension" is transitive, the $C$-morphisms form a category, but this category, being a subcategory of the category of $E$ morphisms, is, by Proposition 1(b), nothing more than a pre-order $\leqslant_{C}$, stronger than $\leqslant_{E}$.

If $A_{1} \prec A_{2} \prec A_{3}$ and $A_{3}$ is a conservative extension of $A_{1}$, then clearly $A_{2}$ is also a conservative extension of $A_{1}$. Thus, in Proposition 1(a), if the given morphisms are $C$-morphisms then so is the factorization. It follows that Proposition 1 remains true if all the $E$ s are changed to $C$ s; in particular, $\leqslant_{C}$ is tree-like.

The reader is invited to translate the definition of $C$-morphism into a purely combinatorial statement (making no reference to ultraproducts) and to check directly that it implies the corresponding translation of the definition of $E$ morphism (fiberwise bounded implies fiberwise constant).

The last of the orderings we wish to consider is the Rudin-Frolik order [4], [5], [12]. Suppose $E, F_{0}, F_{1}, F_{2}, \ldots$ are ultrafilters on $\omega$. Then

$$
E-\sum_{i} F_{i}=\left\{X \subseteq \omega \times \omega \mid\left\{x \mid\{y \mid\langle x, y\rangle \in X\} \in F_{x}\right\} \in E\right\}
$$

is an ultrafilter on $\omega \times \omega$, and $p_{1}\left(E-\Sigma_{i} F_{i}\right)=E$. A morphism of the form $\left[p_{1}\right]_{D}: D \rightarrow E$, where $D=E-\sum_{i} F_{i}$ for some $F_{i}$, will be called a RudinFrolik morphism, or $R F$-morphism, in standard form. Any composite $D \cong D^{\prime}$ $\rightarrow E$ of an isomorphism and an $R F$-morphism in standard form will be called an $R F$-morphism. It was shown by Lascar [9] that any $R F$-morphism is a $C$ morphism. Indeed, if the $R F$-morphism is in standard form, which we may assume without loss of generality, then, for any parametrically definable subset $S=\left\{\left.x\right|^{*} R(x, q)\right\}$ of $\left(E-\sum_{i} F_{i}\right)$-prod $N$, we have $\hat{p}_{1}^{-1}(S)=\{x$ $\in E$-prod $\left.\left.\left.N\right|^{*} P(x \text {, [identity }]_{E}\right)\right\}$, where the predicate $P(a, b)$ on $\omega$ is defined as $\{j \mid R(a, g(b, j))\} \in F_{b}$ and $g: \omega \times \omega \rightarrow \omega$ is chosen so that $[g]=q$. Thus, $\hat{p}_{1}^{-1}(S)$ is parametrically definable in $E$-prod $N$, which suffices to show that $\left[p_{1}\right]$ is a $C$-morphism. 
The formula $\left(D-\Sigma_{i} E_{i}\right)-\sum_{i j} F_{i j} \cong D-\Sigma_{i}\left(E_{i}-\sum_{j} F_{i j}\right)$ shows that the composite of two $R F$-morphisms is an $R F$-morphism; if $F_{i}$ are principal, then [ $\left.p_{1}\right]: E-\Sigma_{i} F_{i} \cong E$, so the identity is an $R F$-morphism. Thus, $R F$-morphisms form a category, a subcategory of the category of $C$-morphisms by Lascar's result, and thus simply a pre-order $\leqslant_{R F}$. The analog of Proposition 1, with $R F$ in place of $E$, was proved by Rudin [12]; in particular, $\leqslant_{R F}$ is treelike.

In the chain of implications

$$
\leqslant_{R F} \rightarrow \leqslant_{C} \rightarrow \leqslant_{E} \rightarrow \leqslant_{R K},
$$

the last implication cannot be reversed. Indeed, Kunen's theorem [7] that $\leqslant_{R K}$ is not linear and the well-known fact that $\leqslant_{R K}$ is directed upward imply that $\leqslant_{R K}$ is not tree-like. (The weaker result that not every morphism is an $E$ morphism is much easier to prove and does not depend on Kunen's theorem.) We shall show in this paper that neither of the other implications is reversible if the continuum hypothesis holds. In the case of the first implication, this answers a question of Lascar [9].

We close this introductory section with a few remarks about the preordering ᄃ terms as follows: $E \subseteq D$ if there is a morphism $[f]_{D}: D \rightarrow E$ such that $\hat{f}(E-\operatorname{prod} N)$ is either all of $D$-prod $N$ or a noncofinal subset of $D$-prod $N$. Clearly, $ᄃ$ fits between $\leqslant_{E}$ and $\leqslant_{R K}$ in the chain of implications. It is distinct from $\leqslant_{E}$ because it is directed upward, and it is distinct from $\leqslant_{R K}$ if the continuum hypothesis holds, for then there are 드-minimal nonprincipal ultrafilters that are not $\leqslant_{R K}$ minimal [12].

2. Ultrafilters over ultraproducts. Let $D$ be an ultrafilter on $\omega$, and let ${ }^{*} V$ be the ultrapower of $V$ with respect to $D$. Recall that any morphism with codomain $D$ is, up to isomorphism, of the form $\left[p_{1}\right]_{E}: E \rightarrow D$ for some ultrafilter $E$ on $\omega \times \omega$. For any given $E,\left[p_{1}\right]_{E}$ has codomain $D$ iff $E$ extends the filter $D \times \omega=\{X \times \omega \mid X \in D\}$. Thus, to study morphisms to $D$ it suffices to study ultrafilters extending $D \times \omega$. In this section, we shall define a canonical correspondence between such ultrafilters and ultrafilters in the Boolean algebra $* \mathcal{P}(\omega)$ of internal subsets of $* \omega$.

For any $A \subseteq \omega \times \omega$, we define a function $\tilde{A}: \omega \rightarrow \mathcal{P}(\omega)$ by $\tilde{A}(x)=\{y \mid\langle x, y\rangle$ $\in A\}$; thus, we have $[\tilde{A}] \in{ }^{*} \mathscr{P}(\omega)$. (We simplify notation by omitting the subscript from $[\tilde{A}]_{D}$.) It is obvious that by sending $A$ to $[\tilde{A}]$ we have a surjective homomorphism of Boolean algebras, $\mathscr{P}(\omega \times \omega) \rightarrow * \mathscr{P}(\omega)$, whose kernel is the ideal dual to $D \times \omega$. Therefore, there is a bijection between ultrafilters in $\mathscr{P}(\omega \times \omega)$ that extend $D \times \omega$ and ultrafilters in $* \mathscr{P}(\omega)$; we write $E / D$ for the ultrafilter in $* \mathscr{P}(\omega)$ corresponding to $E$. Thus, $A \in E$ iff $[\tilde{A}] \in E / D$. We note that $\left[p_{1}\right]_{E}$ is an isomorphism from $E$ to $D$ iff $E / D$ is principal. 
Proposition 2. Let $E$ be an ultrafilter extending $D \times \omega$. Then $\left[p_{1}\right]_{E}: E \rightarrow D$ is an E-morphism iff the intersection of $E / D$ with any ${ }^{*}$ finite subset of $* \mathscr{T}(\omega)$ is internal.

Proof. Since every *finite subset of $* \mathcal{T}(\omega)$ generates a *finite Boolean subalgebra of $* \mathscr{P}(\omega)$, and since the intersection of two internal sets is internal, the proposition is unchanged if we replace "*finite subset" with "*finite Boolean subalgebra". The intersection $U$ of $E / D$ with such an algebra $B$ is then an ultrafilter in $\mathfrak{B}$. As such, it is internal iff it is principal (i.e., consists of all supersets in $\mathfrak{B}$ of one of the atoms of $\mathscr{B}$ ); indeed, it is trivial that principal implies internal, while the converse is simply the translation into ${ }^{*} V$ of the fact that, in $V$, all ultrafilters in finite Boolean algebras are principal. Let $\mathcal{Q}$ be the family of atoms of $\mathscr{B}$, a * finite partition of ${ }^{*} \omega$. For $U$ to be principal means precisely that some member of $\mathscr{Q}$ is in $U$. As any *finite partition of ${ }^{*} \omega$ is the set of atoms of a *finite Boolean algebra, the last clause of the proposition can be equivalently expressed as "every * finite partition of * $\omega$ contains a set in $E / D$ ", or as "every internal map from * $\omega$ into any $n \in{ }^{*} \omega$ is constant on some set of $E / D$ ".

An internal map $[f]_{D}$ from ${ }^{*} \omega$ into ${ }^{*} \omega$ is the germ modulo $D$ of a map $f: \omega \rightarrow{ }^{\omega} \omega$; associate to it the map $g: \omega \times \omega \rightarrow \omega$ defined by $g(x, y)=f(x)(y)$. Observe that $[f]_{D}$ maps ${ }^{*} \omega$ into an element $[h]_{D}$ of ${ }^{*} \omega$ (where $h: \omega \rightarrow \omega$ ) iff, for $D$-almost all $x$, for all $y, f(x)(y)<h(x)$, that is, iff $g$ is fiberwise bounded by $h$ on a set of $D \times \omega$. Redefining $f$ on a set not in $D$, we may, without affecting $[f]_{D}$, assume that $g$ is fiberwise bounded on all of $\omega \times \omega$. For $[f]_{D}$ to be constant on a set $[\tilde{A}]$ of $E / D$ is the same as for $g$ to be fiberwise constant on $A \cap(B \times \omega)$ for some $B \in D$ (so $B \times \omega \in E)$. Thus, the last clause of the proposition can be equivalently expressed as "every fiberwise bounded function on $\omega \times \omega$ is fiberwise constant on some set of $E$." But we saw in $\S 1$ that this is equivalent to $\left[p_{1}\right]_{E}$ being an end-extension morphism.

Proposition 3. Let $E$ be an ultrafilter extending $D \times \omega$. Then $\left[p_{1}\right]_{E}: E \rightarrow D$ is a C-morphism iff the intersection of $E / D$ with any ${ }^{*}$ countable subset of $* \mathscr{P}(\omega)$ is internal.

Proof. Suppose $\left[p_{1}\right]_{E}$ is a $C$-morphism, and let $F$ be an internal map of ${ }^{*} \omega$ into $* \mathscr{P}(\omega)$. To show that the intersection of $E / D$ with the range of $F$ is internal, it suffices to show that $\left\{[h]_{D} \in{ }^{*} \omega \mid F\left([h]_{D}\right) \in E / D\right\}$ is parametrically definable in ${ }^{*} N$, so, because $\left[p_{1}\right]_{E}$ is a $C$-morphism, it suffices to show that this set is the pre-image under $\hat{p}_{1}$ of a parametrically definable subset of $E$-prod $N$. To do this, observe that the binary relation $y^{*} \in F(z)$ on ${ }^{*} \omega$, being internal (because $F$ is internal), must be $[\tilde{Q}]_{D}$ for some $Q \subseteq \omega \times(\omega \times \omega)$. (Recall that we have identified $\omega \times \omega$ with $\omega$, so $\tilde{Q}$ is defined; $\tilde{Q}(x)=\{\langle y, z\rangle \mid\langle x, y, z\rangle$ $\in Q\}$.) For each $h: \omega \rightarrow \omega$, define $Q_{h} \subseteq \omega \times \omega$ to be 


$$
Q_{h}=\{\langle x, y\rangle \mid\langle x, y, h(x)\rangle \in Q\} .
$$

For any $l: \omega \rightarrow \omega$, we have the following chain of equivalences:

$$
\begin{aligned}
{[l]_{D} \in F\left([h]_{D}\right) } & \text { iff }\left\langle[l]_{D},[h]_{D}\right\rangle \in[\tilde{Q}]_{D} \\
& \text { iff }\{x \mid\langle x, l(x), h(x)\rangle \in Q\} \in D \\
& \text { iff }\left\{x \mid\langle x, l(x)\rangle \in Q_{h}\right\} \in D \\
& \text { iff }[l]_{D} \in\left[\tilde{Q}_{h}\right]_{D} .
\end{aligned}
$$

Thus, $F\left([h]_{D}\right)=\left[\tilde{Q}_{h}\right]_{D}$, and we have

$$
\begin{aligned}
F\left([h]_{D}\right) \in E / D & \text { iff }\left[\tilde{Q}_{h}\right] \in E / D \\
& \text { iff } Q_{h} \in E \\
& \text { iff }\left\{t \in \omega \times \omega \mid Q\left(p_{1}(t), p_{2}(t), h p_{1}(t)\right)\right\} \in E \\
& \text { iff } E \text {-prod } N \text { satisfies } Q\left(\left[p_{1}\right]_{E},\left[p_{2}\right]_{E}, \hat{p}_{1}[h]_{D}\right) \\
& \text { iff }[h] \in \hat{p}_{1}^{-1}\{z \in E-\operatorname{prod} N \mid
\end{aligned}
$$

$E$-prod $N$ satisfies $\left.Q\left(\left[p_{1}\right]_{E},\left[p_{2}\right]_{E}, z\right)\right\}$.

As the set to which $\hat{p}_{1}^{-1}$ is applied in the last clause is parametrically defined, we have completed the proof that $E / D$ intersects the range of $F$ in an internal set.

Conversely, assume that $E / D$ intersects every *countable subset of *⿻P $(\omega)$ in an internal set, and consider any parametrically definable $X \subseteq E$-prod $N$; we must show that $\hat{p}_{1}^{-1}(X)$ is parametrically definable in ${ }^{*} N$, or, equivalently, that it is internal in ${ }^{*} V$. We may assume, without loss of generality, that $X$ is of the form

$$
\left\{z \in E-\operatorname{prod} N \mid E-\operatorname{prod} N \text { satisfies } Q\left(\left[p_{1}\right]_{E},\left[p_{2}\right]_{E}, z\right)\right\}
$$

for some $Q \subseteq \omega \times \omega \times \omega$, for the parameters that occur in any definition of $X$ can be defined from $\left[p_{1}\right]_{E}$ and $\left[p_{2}\right]_{E}$. Using this $Q$, define $Q_{h}$ for $h: \omega \rightarrow \omega$ as in the first part of this proof, and define $F:^{*} \omega \rightarrow{ }^{*} \mathscr{P}(\omega)$ by setting $y^{*} \in F(z)$ iff $\langle y, z\rangle \in[\tilde{Q}]_{D}$. Then $F$ is internal, and the chains of equivalences in the first part of the proof are still correct. Thus, we have

$$
\begin{aligned}
{[h]_{D} \in \hat{p}_{1}^{-1}(X) } & \text { iff } F\left([h]_{D}\right) \in E / D \\
& \text { iff }[h]_{D} \in F^{-1}(E / D \cap \text { Range of } F) .
\end{aligned}
$$


As $F$ is internal, its range is *countable, so this range intersects $E / D$ in an internal set. Using once more the fact that $F$ is internal, we see that $\hat{p}_{1}^{-1}(X)$ is internal, as required.

Proposition 4. Let $E$ be an ultrafilter extending $D \times \omega$. Then $\left[p_{1}\right]_{E}: E \rightarrow D$ is an $R F$-morphism if and only if $E / D$ is internal.

Proof. We show first that, if $\left[p_{1}\right]_{E}$ is an $R F$-morphism, then it is an $R F$ morphism in standard form. The assumption is that $\left[p_{1}\right]_{E}$ is the composite of an isomorphism $[f]_{E}: E \rightarrow E^{\prime}=D-\sum_{i} F_{i}$ and the projection $\left[p_{1}\right]_{E^{\prime}}: E^{\prime}$ $\rightarrow D$. Thus $p_{1} \circ f$ and $p_{1}$ are equal on a set in $E$; modifying $f$ on the complement of this set, we may arrange, without changing $[f]_{E}$, that $p_{1} \circ f=p_{1}$. So $f$ has the form $f\langle x, y\rangle=\left\langle x, f_{x}(y)\right\rangle$ for certain functions $f_{x}$. As $[f]_{E}$ is an isomorphism, $f$ may be assumed to be a permutation of $\omega \times \omega$; then each $f_{x}$ is also a permutation, and we may define the ultrafilters $G_{x}=f_{x}^{-1}\left(F_{x}\right)$. It is easy to verify that

$$
E=f^{-1}\left(E^{\prime}\right)=f^{-1}\left(D-\sum_{i} F_{i}\right)=D-\sum_{i} G_{i},
$$

so $\left[p_{1}\right]_{E}$ is an $R F$-morphism in standard form, as claimed. Therefore, in proving the proposition, we may assume that it refers to $R F$-morphisms in standard form.

For $E / D$ to be an internal ultrafilter in $* \mathscr{P}(\omega)$ means that there is a function $F$, defined on $\omega$ and taking ultrafilters in $\mathscr{P}(\omega)$ as values, such that $E / D$ $=[F]_{D}$. This means that for all $A \subseteq \omega \times \omega$,

$$
\begin{aligned}
A \in E & \text { iff }[\tilde{A}] \in[F] \\
& \text { iff }\{x \mid \tilde{A}(x) \in F(x)\} \in D \\
& \text { iff } A \in D-\sum_{i} F(i),
\end{aligned}
$$

so $E=D-\sum_{i} F_{i}$ and $\left[p_{1}\right]_{E}$ is an $R F$-morphism in standard form. The same steps in reverse order prove the converse.

Notice that the three propositions proved in this section give an alternate proof of the implications between $\leqslant_{R F}$, $\leqslant_{C}$, and $\leqslant_{E}$.

3. Nonconservative end extensions. Phillips has constructed [11] nonconservative elementary extensions (necessarily end extensions) of the standard model of Peano arithmetic. His construction depended on the fact that the language of Peano arithmetic is countable, and this dependence is unavoidable since the standard model $N$ of full arithmetic obviously has only conservative extensions. We shall show in this section that certain nonstandard models ${ }^{*} N$ of full arithmetic, including all ultrapowers of $N$ with respect to nonprincipal 
ultrafilters on $\omega$, have nonconservative end extensions.

THEOREM 5. Let $* V$ be a nonstandard model of $V$, and let ${ }^{*} N$ be the nonstandard model of $N$ consisting of ${ }^{*} \omega$ and its standard relations and functions in ${ }^{*} V$. Let $\kappa$ be the cardinality of ${ }^{*} N$, and assume ${ }^{*} V$ is $\kappa$-saturated. Then ${ }^{*} N$ has a nonconservative end extension.

Before proving this theorem, we introduce some terminology and establish a lemma. Let $T$ be the usual binary tree consisting of finite sequences of zeros and ones; if $s \in T$ and $n \in \omega$, equations involving $s_{n}$ are to be construed as implying that $s$ is long enough for $s_{n}$ to be defined. Paths through the tree $T$ correspond canonically to points in the product space $2^{\omega}$ of countably many copies of $\{0,1\}=2$. We give 2 the discrete topology and $2^{\omega}$ the product topology, so $2^{\omega}$ is homeomorphic to the Cantor set. If $X$ is any subset of $T$, we define a path through $X$ to be a path through $T$ such that infinitely many of the nodes on the path lie in $X$. We call $X$ large if the set of paths through $X$ contains a perfect (i.e., nonempty, closed, without isolated points) subset of $2^{\omega}$. As the paths through $X$ are easily seen to form a Borel (in fact $G_{\delta}$ ) subset of $2^{\omega}, X$ is large iff there are uncountably many paths through it $[8, \mathrm{p} .447]$.

LEMMA. (a) If a large set is partitioned into finitely many pieces, then at least one of the pieces is large.

(b) If $X$ is large, then there is an $n \in \omega$ such that both $\left\{s \in X \mid s_{n}=0\right\}$ and $\{s$ $\in X \mid s_{n}=1$ ) are large.

Proof. (a) Any path through the given large set is obviously also a path through at least one of the pieces (because there are only finitely many pieces). As the original set has uncountably many paths through it, so does one of the pieces.

(b) If the conclusion is false, then for each $n \in \omega$ there is $i_{n}=0$ or 1 such that the set $C_{n}$ of paths through $X$ with $n$th term $i_{n}$ is countable. If $p$ is the path with $n$th term $1-i_{n}$ for all $n$, then every path through $X$ is in the countable set $\cup_{n \in \omega} C_{n} \cup\{p\}$, contrary to the assumption that $X$ is large.

In the following proof, we assume that $T$ and $\omega$ have been identified via some bijection.

Proof of Theorem 5. By our assumption that $V$ is big enough to contain all the sets we need, we have in $V$ the family $L$ of large subsets of $T$. Members of ${ }^{*} L$ in ${ }^{*} V$ will be called *large. We intend to construct an ultrafilter $E$ in the Boolean algebra of parametrically definable subsets of ${ }^{*} T={ }^{*} \omega$ such that $E$ consists of *large sets and the following requirements are satisfied. For each bounded (below ${ }^{*} \omega$ ) parametrically definable unary function $f$ on ${ }^{*} N$,

(1f) there is an $n \in{ }^{*} \omega$ such that $f^{-1}\{n\} \in E$, and, for each parametrically definable subset $A$ of ${ }^{*} N$,

(2A) there is an $n \in{ }^{*} \omega$ such that either $n \in A$ and $\left\{s \in{ }^{*} T \mid s_{n}=0\right\} \in E$ or $n \notin A$ and $\left\{s \in{ }^{*} T \mid s_{n}=1\right\} \in E$. 
To construct such an $E$, we proceed inductively. Note first that the cardinality $\kappa$ of ${ }^{*} N$ is at least the power of the continuum (for there are continuum many almost disjoint subsets of $\omega$, and their extensions in a nonstandard model contain distinct nonstandard elements), so the number of parametrically definable functions and relations on ${ }^{*} N$ is $\kappa$ and we may assume that the requirements $(1 f)$ and $(2 A)$ have been enumerated in a $\kappa$ sequence. We define a decreasing sequence of *large (internal) sets $E_{\alpha}(\alpha<\kappa)$ by induction on $\alpha$ as follows. Let $E_{0}={ }^{*} T$. At limit ordinals $\lambda$, let $E_{\lambda}$ be any *large set included in $E_{\alpha}$ for all $\alpha<\lambda$; such a set exists because of the assumption that ${ }^{*} V$ is $\kappa$-saturated. If $E_{\alpha}$ has been defined and the $\alpha$ th requirement is (1f), let $E_{\alpha+1}$ be a "large subset of $E_{\alpha}$ on which $f$ is constant; such a set exists by the translation into ${ }^{*} V$ of the first part of the lemma. Finally, if $E_{\alpha}$ has been defined and the $\alpha$ th requirement is $(2 A)$, use the translation into ${ }^{*} V$ of the second part of the lemma to find $n \in{ }^{*} \omega$ such that both $\left\{s \in E_{\alpha} \mid s_{n}=0\right\}$ and $\left\{s \in E_{\alpha} \mid s_{n}=1\right\}$ are *large. Let $E_{\alpha+1}$ be the first or second of these sets according to whether or not $n \in A$.

Define $E$ to be the collection of parametrically definable subsets of ${ }^{*} \omega$ (identified with ${ }^{*} T$ ) that contain $E_{\alpha}$ for some $\alpha<\kappa$. Since the sets required to be in $E$ by requirements $(1 f)$ and $(2 A)$ are parametrically definable, the construction of the $E_{\alpha}$ 's guarantees that $E$ satisfies these requirements. Applying ( $1 f$ ) to functions $f$ taking only the values 0 and 1 , we see that $E$ is an ultrafilter.

By compactness, there is an elementary extension $M$ of ${ }^{*} N$ containing an element $s$ such that, for all formulas $\Phi(v, \mathbf{q})$ with parameters from ${ }^{*} N, M$ satisfies $\Phi(s, \mathbf{q})$ iff $\left\{x \in{ }^{*} N \mid \Phi(x, \mathbf{q})\right\} \in E$. Since full arithmetic has built-in Skolem functions, we may replace $M$ with the Skolem hull of ${ }^{*} N \cup\{s\}$ and thus assume that every element of $M$ is $f(s, q)$ for some standard $f$ and some $\mathbf{q} \in{ }^{*} N$.

We show that $M$ is an end extension of ${ }^{*} N$. Suppose an element $f(s, \mathbf{q})$ of $M$ is majorized by some $b \in{ }^{*} N$. Let $g$ be the parametrically definable function on $M$ whose value at $x$ is $f(x, q)$ if this is below $b$ and 0 otherwise. As the parameters $q$ and $b$ in the definition of $g$ lie in ${ }^{*} N$, the restriction $\left.g\right|^{*} N$ is a bounded (by $b$ ) parametrically definable function on ${ }^{*} N$. By requirement (1 $\left.\left.g\right|^{*} N\right), E$ contains $\left\{x \in{ }^{*} N \mid g(x)=a\right\}$ for some $a \in{ }^{*} N$. Thus, $g(s)=a$. But, as $f(s, \mathbf{q})<b$, we have $f(s, \mathbf{q})=g(s)=a \in{ }^{*} N$.

We show that $M$ is not a conservative extension of ${ }^{*} N$ by showing that the set $A=\left\{n \in{ }^{*} N \mid s_{n}=1\right\}$, which is the intersection of ${ }^{*} N$ with a set parametrically definable in $M$ from $s$, is not parametrically definable in ${ }^{*} N$. Indeed, if it were parametrically definable, requirement $(2 A)$ would provide an $n \in{ }^{*} \omega$ such that 


$$
n \in A \Rightarrow\left\{x \in{ }^{*} N \mid x_{n}=0\right\} \in E \Rightarrow s_{n}=0,
$$

and similarly $n \notin A \Rightarrow s_{n}=1$, contradicting in either case the definition of $A$. Therefore, $M$ is a nonconservative end extension of ${ }^{*} N$.

Let $D$ be a nonprincipal ultrafilter on $\omega$, and let ${ }^{*} V$ be the ultrapower $D$ prod $V$. The associated model of arithmetic is ${ }^{*} N=D$-prod $N$. By a wellknown theorem of Keisler [6], ${ }^{*} V$ is $\kappa_{1}$-saturated. ${ }^{*} N$ has the cardinality of the continuum so, if we assume the continuum hypothesis $(\mathrm{CH})$ the assumptions of Theorem 5 are satisfied, and we conclude that $D$-prod $N$ has a nonconservative end extension $M$. We can arrange for $M$ to be generated by a single element that codes the generator [identity] ${ }_{D}$ of $D$-prod $N$ and the parameter from $M$ needed to define some set whose intersection with $D$-prod $N$ is not parametrically definable in $D$-prod $N$. Thus, $M$ is isomorphic to $E$-prod $N$ for some ultrafilter $E$ on $\omega$, and we obtain the following corollaries.

COROLlARY 6. $(\mathrm{CH})$ Every nonprincipal ultrafilter on $\omega$ is the codomain of an E-morphism that is not a C-morphism.

Corollary 7. (CH) The orderings $\leqslant_{E}$ and $\leqslant_{C}$ do not coincide.

Proof. Use the preceding corollary, the fact that all $C$-morphisms are $E$ morphisms, and Proposition 1(b).

COROLlaRY 8. $(\mathrm{CH})$ There are models $A$ and $B$ of full arithmetic and a type $\tau$ over $A$ such that $B$ is an end extension of $A, \tau$ can be realized in an end extension of $A$, but $\tau$ cannot be realized in an end extension of $B$.

Proof. Let $A$ be $D$-prod $N$ for some nonprincipal ultrafilter $D$ on $\omega$. Let $\tau$ be the type over $A$ determined by an ultrafilter $E$ that satisfies the requirements in the proof of Theorem 5. Thus, as was shown in the proof, $\tau$ can be realized in an end extension of $A$, but not in a conservative extension of $A$. Let $B$ be any proper conservative extension of $A$. Then any end extension of $B$ is necessarily a conservative extension of $A$ (see [2, Lemma 5c]) and therefore cannot realize $\tau$.

If $A, B$, and $\tau$ are as in Corollary 8, then there are extensions of $B$ containing an element $s$ realizing $\tau$, such that $s$, as well as any element not in $A$ but definable from $s$ and members of $A$, exceeds all members of $B$ (see [3, Theorem 3]). However, by Corollary 8 , there will be elements, not in $B$ but definable from $s$ and members of $B$, that are majorized by members of $B$.

It is not difficult to prove the following strengthening of the first part of the lemma in the proof of Theorem 5.

LEMMA. Any function defined on a large set is constant or one-to-one on a large subset. 
Using this lemma (or rather its translation into ${ }^{*} V$ ), we can arrange that, in the proof of Theorem 5, each parametrically definable $f$ on ${ }^{*} N$ is one-to-one or constant on some $E_{\alpha}$. If all internal subsets of ${ }^{*} \omega$ are parametrically definable in ${ }^{*} N$ (for example, if ${ }^{*} V=D$-prod $V$ for some ultrafilter on $\omega$ ), then all the $E_{\alpha}$ 's are in $E$. It is then easy to check that the Skolem hull $M$ of ${ }^{*} N \cup\{s\}$ (where $s$ realizes the type given by $E$, as in the proof of Theorem 5) is a minimal extension of ${ }^{*} N$, i.e., no proper submodel of $M$ properly includes ${ }^{*} N$. Translating this result into the language of ultrafilters, we have the following corollary.

COROLlaRY 9. (CH) Every nonprincipal ultrafilter $D$ on $\omega$ is the codomain of a nonconservative E-morphism that cannot be factored except by isomorphisms.

In Corollary 9, let us take $D$ to be a nonprincipal ultrafilter minimal in the $R K$ ordering, hence also in the $E, C$, and $R F$ orderings. (Such $D$ 's, called Ramsey ultrafilters, are easily constructed using the continuum hypothesis.) Let $[f]_{E}: E \rightarrow D$ be a nonconservative $E$-morphism that cannot be nontrivially factored. Clearly, $E$ is not minimal (among nonprincipal ultrafilters) in $\leqslant_{E}$, for $D$ is strictly below it. However, $E$ is minimal (among nonprincipal ultrafilters) in $\leqslant_{C}$. For suppose $[g]_{E}: E \rightarrow F$ is a nonisomorphic $C$-morphism. If $[f]_{E}$ factored through $[g]_{E}$, we would have an immediate contradiction to the assumed properties of $[f]_{E}$. So by Proposition $1(\mathrm{a}),[g]_{E}=[h]_{D}$ $\cdot[f]_{E}$ for some $[h]_{D}: D \rightarrow F$. As $[g]_{E}$ is conservative and $[f]_{E}$ is not, $[h]_{D}$ is not an isomorphism. By choice of $D$, it follows that $F$ is principal. This proves

COROLLARY 10. $(\mathrm{CH})$ There are nonprincipal ultrafilters minimal in $\leqslant_{C}$ but not minimal in $\leqslant_{E}$.

We remark that the family of large sets, as defined in the proof of Theorem 5 , is the greatest family making the lemma in that proof true. There are other families making that lemma, as well as the lemma to Corollary 9, true; any such family would have worked just as well in our proofs. For example, we could have used the family of subsets of $T$ on which the functions $s \mapsto s_{n}$, for sufficiently large $n \in{ }^{*} \omega$, are independent; our proof would then bear a closer resemblance to Phillips's construction [11] of a minimal nonconservative extension of the standard model of Peano arithmetic.

4. A conservative non- $R F$ morphism. Let $D$ be a nonprincipal ultrafilter on $\omega$, and let ${ }^{*} V$ be the ultrapower of $V$ with respect to $D$. Throughout this section, we assume the continuum hypothesis and the existence of an external subset $A$ of $\aleph_{1}$ such that $A \cap \alpha$ is internal for all $\alpha \in{ }^{*} \aleph_{1}$. (More precisely, $A \cap\left\{x \mid x^{*}<\alpha\right\}$ is internal whenever $\alpha^{*} \in{ }^{*} \aleph_{1}$.) Using these assumptions, we construct a conservative non- $R F$ morphism with codomain $D$. The subset $A$ of 
${ }^{*} \aleph_{1}$ whose existence is assumed will be constructed, without appeal to the continuum hypothesis, in the next section.

Consider the standard inductive procedure for constructing a nonprincipal ultrafilter on $\omega$, using a well-ordering of $\mathscr{P}(\omega)$. One constructs a sequence of filters, beginning with the filter of cofinite subsets of $\omega$, taking unions at limit stages, and, at any successor stage $\alpha+1$, adjoining the $\alpha$ th subset of $\omega$ (in the well-ordering) unless its complement is already in the filter. (To adjoin a set to a filter means to add all supersets of the intersections of this set with sets in the filter, so the result is again a filter.) This procedure obviously produces an ultrafilter no later than stage $\lambda$, where $\lambda$ is the length of the well-ordering of $\mathscr{P}(\omega)$. As we are assuming the continuum hypothesis, we may assume $\lambda=\aleph_{1}$, and then we obtain an ultrafilter precisely at stage $\aleph_{1}$, for no nonprincipal ultrafilter has a countable base.

The construction just described gives, at each successor stage $\alpha+1$, preference to the $\alpha$ th subset of $\omega$ as opposed to its complement, for the $\alpha$ th subset is put into the filter whenever possible. One could, of course, change the construction to give the same sort of preference to the complement of the $\alpha$ th set at stage $\alpha+1$. More generally, given any subset $B$ of $\kappa_{1}$, one can change the construction so that, the $\xi$ th time one has a choice between adjoining a set and adjoining its complement (i.e., neither the set nor its complement is yet in the filter) one adjoins the set under consideration if $\xi \in B$ and one adjoins the complement if $\xi \notin B$. This procedure leads, in $\aleph_{1}$ steps, to an ultrafilter that "codes" $B$. (Here, and in what follows, we assume that we have fixed, once and for all, a well-ordering of $\mathscr{P}(\omega)$ of length $\aleph_{1}$.) For $B \subseteq \aleph_{1}$ and $\beta \leqslant \aleph_{1}$, let $F(B, \beta)$ be the filter obtained by following this procedure up to, but not including, the $\beta$ th choice. Thus, $F(B, \beta)$ is an ultrafilter iff $\beta=\kappa_{1}$, and, for $\beta<\aleph_{1}, F(B, \beta)=F(B \cap \beta, \beta)$. We collect for future reference a number of obvious facts about the function $F$.

(1) If $\beta \leqslant \alpha \leqslant \aleph_{1}$, then $F(B \cap \beta, \beta) \subseteq F(B, \alpha)$, with equality iff $\beta=\alpha$.

(2) If $B \cap \beta \neq B^{\prime} \cap \beta$, then $F(B, \beta)$ and $F\left(B^{\prime}, \beta\right)$ are incompatible (i.e., their union contains two disjoint sets).

(3) If $S$ is the $\beta$ th subset of $\omega$ (in the fixed well-ordering) and $\beta<\alpha$, then $F(B, \alpha)$ contains $S$ or $\omega-S$.

(4) Every ultrafilter on $\omega$ is $F\left(B, \aleph_{1}\right)$ for some (unique) $B \subseteq \aleph_{1}$.

We now use $F$ to construct a conservative non- $R F$ morphism to $D$. By Propositions 3 and 4, it is necessary and sufficient to construct an external ultrafilter $E$ in $* \mathscr{P}(\omega)$ whose intersection with every * countable subset of $* \mathscr{P}(\omega)$ is internal. Let $A \subseteq{ }^{*} \aleph_{1}$ be external but such that $A \cap \alpha$ is internal for all $\alpha<{ }^{*} \aleph_{1}$. Then, for such $\alpha, E_{\alpha}={ }^{*} F(A \cap \alpha, \alpha)$ is an internal filter in the Boolean algebra $* \mathscr{P}(\omega)$. Translating (1) into ${ }^{*} V$, we find that $E_{\beta} \subseteq E_{\alpha}$ for $\beta \leqslant \alpha \leqslant{ }^{*} \aleph_{1}$. Thus $E=\cup_{\alpha<^{*} \aleph_{1}} E_{\alpha}$ is also a filter in ${ }^{*} \mathcal{P}(\omega)$. The translation 
into ${ }^{*} V$ of (3) implies that $E$ is an ultrafilter in $* \mathcal{P}(\omega)$ and that its intersection with any *countable (hence internal) $C$ is the internal set $C \cap E_{\alpha}$ for any sufficiently large $\alpha$. It remains to show that $E$ is external. Suppose it were internal. By the translation into ${ }^{*} V$ of $(4)$, it would be ${ }^{*} F\left(B,{ }^{*} \aleph_{1}\right)$ for some internal $B \subseteq{ }^{*} \aleph_{1}$. For any $\alpha<{ }^{*} \aleph_{1}, E$ would be an extension of ${ }^{*} F(B, \alpha)$ (by the translation of (1)) as well as of $E_{\alpha}={ }^{*} F(A \cap \alpha, \alpha)$. By the translation of (2) into ${ }^{*} V$, we could infer that $A \cap \alpha=B \cap \alpha$, and, since $\alpha$ was arbitrary, $A=B$, which contradicts the fact that $A$ is external. Therefore, $E$ is external, as required.

Summarizing, and anticipating the proof that the required $A$ exists, we have the following result analogous to Corollary 6.

THEOREM 11. (CH) Every nonprincipal ultrafilter on $\omega$ is the codomain of a $C$ morphism that is not an RF-morphism.

COROLlaRY 12. $(\mathrm{CH})$ The orderings $\leqslant_{C}$ and $\leqslant_{R F}$ are distinct.

If, in the standard inductive method of constructing ultrafilters that led to the function $F$, we insert more steps to guarantee that every function on $\omega$ is constant or one-to-one on a set of the filter, then (we have the standard construction of a Ramsey ultrafilter and) we are led to a different function $F^{\prime}$ which has all the properties of $F$ that we used in the proof of Theorem 11 . Carrying out this proof with $F^{\prime}$ in place of $F$, we find that every internal function on ${ }^{*} \omega$ is constant or one-to-one on some set in the external ultrafilter $E$. Thus, we easily get the following analog of Corollary 9.

COROLLARY 13. (CH) Every nonprincipal ultrafilter $D$ on $\omega$ is the codomain of a non-RF C-morphism that cannot be factored except by isomorphisms.

In the reasoning leading from Corollary 9 to Corollary 10, we can replace $\leqslant_{E}$ and $\leqslant_{C}$ by $\leqslant_{C}$ and $\leqslant_{R F}$ respectively and obtain the following consequence of Corollary 13.

COROLlARY 14. $(\mathrm{CH})$ There are nonprincipal ultrafilters minimal in $\leqslant_{R F}$ but not minimal in $\leqslant_{C}$.

5. An external set with internal segments. Again, let $D$ be a nonprincipal ultrafilter on $\omega$, and let ${ }^{*} V$ be the ultrapower of $V$ with respect to $D$. In this section we construct an external subset $A \subseteq{ }^{*} \aleph_{1}$ such that $A \cap \alpha$ is internal for all $\alpha^{*} \in{ }^{*} \boldsymbol{N}_{1}$. Notice that it suffices to consider standard countable ordinals $\alpha$, for these are cofinal in ${ }^{*} \aleph_{1}$ (because $D$ is on a countable set). Thus, we construct an external $A \subseteq{ }^{*} \aleph_{1}$, such that $A \cap{ }^{*} \alpha$ is internal for all $\alpha \in \aleph_{1}$. In fact, we shall first define the internal sets $A \cap{ }^{*} \alpha=\left[A_{\alpha}\right]_{D}$, where $A_{\alpha}: \omega \rightarrow \mathscr{P}(\alpha)$, so that $\left[A_{\alpha}\right]_{D}=\left[A_{\beta} \cap \alpha\right]_{D}$ for $\alpha \leqslant \beta<\kappa_{1}$, and then we shall define $A$ to be the union of these $\left[A_{\alpha}\right]_{D}$ 's. Thus, we need only define 
functions $A_{\alpha}: \omega \rightarrow \mathscr{P}(\alpha)$ for $\alpha<\aleph_{1}$ so that

(1) $\left\{n \in \omega \mid A_{\alpha}(n)=A_{\beta}(n) \cap \alpha\right\}=C_{\alpha \beta} \in D$ for all $\alpha \leqslant \beta$ and

(2) $\cup_{\alpha<\aleph_{1}}\left[A_{\alpha}\right]_{D}=A$ is external.

To define such functions $A_{\alpha}$, we proceed by induction on $\alpha$. To begin the induction let $A_{\alpha}(n)$ be empty for all $\alpha<\omega$ and all $n<\omega$. Suppose that $\gamma \geqslant \omega$, that $A_{\alpha}$ has been defined for all $\alpha<\gamma$, and that (1) holds for all $\alpha \leqslant \beta<\gamma$. Let $g$ be a bijection from $\omega$ onto $\gamma$. For each $n<\omega$, let $k(n)$ be the largest natural number $k \leqslant n$ for which $n \in \cap_{i=0}^{k-1} C_{g(i) g(i+1)}$, where we adopt the convention that if $\alpha>\beta$ then $C_{\alpha \beta}$ means $C_{\beta \alpha}$. Since $D$ contains all the sets $C_{g(i) g(i+1)}$, it also contains $\{n \mid k(n) \geqslant m\}$ for each $m<\omega$. For each $n$, let $\xi(n)$ be the largest of the ordinals $g(i)$ for $0 \leqslant i \leqslant k(n)$. Thus, $A_{g(i)}(n)$ $=A_{\xi(n)}(n) \cap g(i)$ for all $i \leqslant k(n)$. We shall define $A_{\gamma}$ so that, for each $n$,

$$
A_{\gamma}(n) \cap \xi(n)=A_{\xi(n)}(n) .
$$

Then $A_{g(i)}(n)=A_{\gamma}(n) \cap g(i)$ whenever $i \leqslant k(n)$. For each $i$, we have $i \leqslant k(n)$ for $D$-almost all $n$, so (1) holds for $\alpha \leqslant \beta=\gamma$. It remains to define $A_{\gamma}(n)-\xi(n) \subseteq \gamma-\xi(n)$ in such a way as to obtain (2).

Let $\left\{S_{\nu} \mid \nu<\aleph_{1}\right\}$ be an enumeration of $\aleph_{1}$ subsets of $\omega$ such that the symmetric difference $S_{\nu} \Delta S_{\mu}$ is infinite whenever $\nu \neq \mu$, for example, $S_{\nu}$ $=p_{1}^{-1}\left(S_{\nu}^{\prime}\right)$ where the $S_{\nu}^{\prime}$ are distinct. (The $S_{\nu}$ are supposed to be defined once and for all, independently of $\gamma$.) Each ordinal $\alpha$ can be expressed uniquely in the form $\lambda(\alpha)+b(\alpha)$ where $\lambda(\alpha)$ is a limit ordinal or zero and $b(\alpha)<\omega$. We complete the definition of $A_{\gamma}$ by setting

$$
A_{\gamma}(n)-\xi(n)=\left\{\alpha \mid \xi(n) \leqslant \alpha<\gamma \text { and } b(\alpha) \in S_{\gamma}\right\} .
$$

To prove (2), we first consider arbitrary but fixed $n<\omega$ and $B \subseteq \aleph_{1}$, and we define

$$
X=\left\{\alpha<\aleph_{1} \mid \alpha \text { is a limit ordinal and } A_{\alpha}(n)=B \cap \alpha\right\} .
$$

We show first that no member of $X$ is a limit of smaller members of $X$. Indeed, suppose $\beta_{0}<\beta_{1}<\cdots$ were an increasing $\omega$-sequence in $X$ whose limit $\gamma$ is also in $X$. Consider $\xi_{\gamma}(n)$, the $\xi(n)$ which occurs in the $\gamma$ th stage of our induction when $A_{\gamma}$ is being defined. As it is less than $\gamma$, it is below some $\beta_{i}$ which we call simply $\beta$. Let $\xi$ be $\xi_{\gamma}(n)$ or $\xi_{\beta}(n)$ whichever is larger. For any ordinal $\alpha$ above $\xi$ but below $\beta$, we have

$$
\begin{aligned}
& b(\alpha) \in S_{\beta} \text { iff } \alpha \in A_{\beta}(n) \quad \text { (definition of } A_{\beta} \text { ) } \\
& \text { iff } \alpha \in B \quad(\beta \in X) \\
& \text { iff } \alpha \in A_{\gamma}(n) \quad(\gamma \in X) \\
& \text { iff } b(\alpha) \in S_{\gamma} \quad \text { (definition of } A_{\gamma} \text { ). }
\end{aligned}
$$

But as $\alpha$ varies from $\xi$ to $\beta, b(\alpha)$ ranges over a cofinite subset of $\omega$ (as $\beta$ is a limit ordinal by definition of $X$ ), and thus the above chain of equivalences 
contradicts the choice of the sets $S_{\nu}$. Therefore, $X$ contains none of its own limits.

It follows that we can find a closed cofinal subset $Y=Y(B, n)$ of $\aleph_{1}$ such that $A_{\alpha}(n) \neq B \cap \alpha$ for any $\alpha \in Y$. Indeed, if $X$ is bounded below $\aleph_{1}$ we can take $Y$ to consist of all limit ordinals above all the members of $X$, while if $X$ is unbounded in $\aleph_{1}$ we can take $Y$ to consist of all limits of increasing $\omega$ sequences from $X$.

Now we can show that $A=\cup_{\alpha<N_{1}}\left[A_{\alpha}\right]_{D}$ is external by showing that it differs from an arbitrary internal $[B]_{D} \subseteq{ }^{*} \boldsymbol{\aleph}_{1}$, where $B: \omega \rightarrow \mathcal{P}\left(\boldsymbol{\aleph}_{1}\right)$. Given any such $B$, we have, for each $n<\omega$, a closed cofinal subset $Y_{n}=Y(B(n), n) \subseteq \aleph_{1}$ such that

$$
\alpha \in Y_{n} \text { implies } A_{\alpha}(n) \neq B(n) \cap \alpha .
$$

As the intersection of countably many closed cofinal subsets of $\boldsymbol{\kappa}_{1}$ is nonempty, we can find $\alpha \in \bigcap_{n<\omega} Y_{n}$. For such an $\alpha$, (5) implies $\left[A_{\alpha}\right]_{D}$ $\neq[B]_{D} \cap{ }^{*} \alpha$. But it is clear from (1) that $\left[A_{\alpha}\right]_{D}=A \cap{ }^{*} \alpha$. So $A \neq[B]_{D}$, as required.

Addendum. The "perfect set" approach to the construction of nonconservative extensions was independently discovered by $\mathrm{K}$. Potthoff. In a preprint entitled "A simple tree lemma and its application to a counterexample of Phillips", which arrived while the present paper was being typed, Potthoff establishes the two lemmas in $\$ 3$ (with some additional definability information needed because he works with a countable language) and uses them to give an alternate proof of the theorem of Phillips [11].

\section{REFERENCES}

1. A. Blass, Orderings of ultrafilters, Thesis, Harvard Univ., 1970.

2. On certain types and models for arithmetic, J. Symbolic Logic 39 (1974), 151-162.

3. Amalgamation of non-standard models of arithmetic, J. Symbolic Logic (to appear).

4. D. Booth, Ultrafilters on a countable set, Ann. Math. Logic 2 (1970/71), 1-24. MR 43 \#3104.

5. Z. Frolik, Sums of ultrafilters, Bull. Amer. Math. Soc. 73 (1967), 87-91. MR 34 \#3525.

6. H. J. Keisler, Ultraproducts and saturated models, Nederl. Akad. Wetensch., Proc. Sér. A 67 = Indag. Math. 26 (1964), 178-186. MR 29 \#5745.

7. K. Kunen, Ultrafilters and independent sets, Trans. Amer. Math. Soc. 172 (1972), 299-306. MR 47 \#3170.

8. K. Kuratowski, Topology, Vol. I, 5th ed., PWN, Warsaw; Academic Press, New York and London, 1966. MR 36 \#840.

9. D. Lascar, Ultrafiltres et theories stables (to appear).

10. R. G. Phillips, Omitting types in arithmetic and conservative extensions, Victoria Symposium on Nonstandard Analysis (A. Hurd and P. Loeb, Eds.), Lecture Notes in Math., vol. 369, Springer-Verlag, Berlin and New York, 1974, pp. 195-202.

11. - A minimal extension that is not conservative, Michigan Math. J. 21 (1974), 27-32. MR 50 \# 94.

12. M. E. Rudin, Partial orders on the types in $\beta N$, Trans. Amer. Math. Soc. 155 (1971), 353-362. MR 42 \#8459.

Department of Mathematics, University of Michigan, Ann Arbor, Michigan 48109 